\title{
Hidden star clusters in the starburst galaxy He 2-10
}

\author{
William D. Vacca ${ }^{1}$, Kelsey E. Johnson ${ }^{2}$, and Peter S. Conti ${ }^{3}$ \\ ${ }^{1}$ Max-Planck-Institut für Extraterrestrische Physik, \\ Postfach 1312, D-85741 Garching-bei-München, BRD \\ ${ }^{2}$ National Radio Astronomy Observatory, \\ PO Box 0, Socorro, NM 87801, USA \\ ${ }^{3}$ Joint Institute for Laboratory Astrophysics, \\ University of Colorado, Boulder, CO 80309-0440, USA
}

\begin{abstract}
N$-band $(10.8 \mu \mathrm{m})$ imaging of the blue compact starburst galaxy He 2-10 reveals the presence of four emission regions, which have no optical or near-IR counterparts. These sources correspond to the 'ultradense $\mathrm{H}$ II' regions recently identified in $2 \mathrm{~cm}$ and $6 \mathrm{~cm}$ radio maps of this galaxy. We have used the $N$-band data to place constraints on the properties of the stellar clusters, that are deeply embedded within these dust-emitting regions.
\end{abstract}

\section{Introduction}

To better understand the early stages of massive star cluster evolution, we have obtained $J H K^{\prime}$, and $N(10.8 \mu \mathrm{m})$ images of the nuclear region of the blue compact starburst galaxy $\mathrm{He} 2-10(d=9 \mathrm{Mpc})$. The $N$-band images were obtained with OSCIR on the Gemini North telescope and reveal four heavily dust-enshrouded clusters located within 'ultradense H II regions' (UDH II ), discovered in radio maps obtained by Kobulnicky \& Johnson (1999). None of these sources appears in either the optical HST images or the near-IR images (see Figure 1). A more complete description of the observations and results can be found in Vacca et al. (2002).

\section{UDH II region properties in He 2-10 and implications}

Although they have effective radii of only about $10-20 \mathrm{pc}$, the four UDH II regions comprise $>60 \%$ of the total $N$-band flux from He $2-10$, and probably a similar fraction of the total far-IR flux measured by IRAS. The inferred spectral energy distributions of the UDH II regions are very similar to those of Galactic ultracompact $\mathrm{H}$ II regions, and we have modeled the UDH II regions as 'scaledup' versions of these objects. The bolometric luminosities of the UDH II regions are $0.3-2 \times 10^{9} \mathrm{~L}_{\odot}$, and the total mass of dust and gas in each UDH II is 0.2 $1.2 \times 10^{7} \mathrm{M}_{\odot}$. Constraints on the masses and ages of the stellar clusters embedded within the UDH II regions were determined from the observed spectral energy distributions and population synthesis models (see Figure2). All of the stellar clusters enshrouded within the UDH II regions in He 2-10 must have ages $\tau \lesssim 5 \times 10^{6} \mathrm{yr}$ and masses $M \gtrsim 5 \times 10^{5} \mathrm{M}_{\odot}$. Hence, UDH II regions represent the youngest phases of Super Star Clusters, in which the clusters are still deeply embedded in their birth clouds. The logarithmic ratios of the radio to far-IR flux densities for the individual UDH II regions, and He 2-10 as a whole, are signifi- 

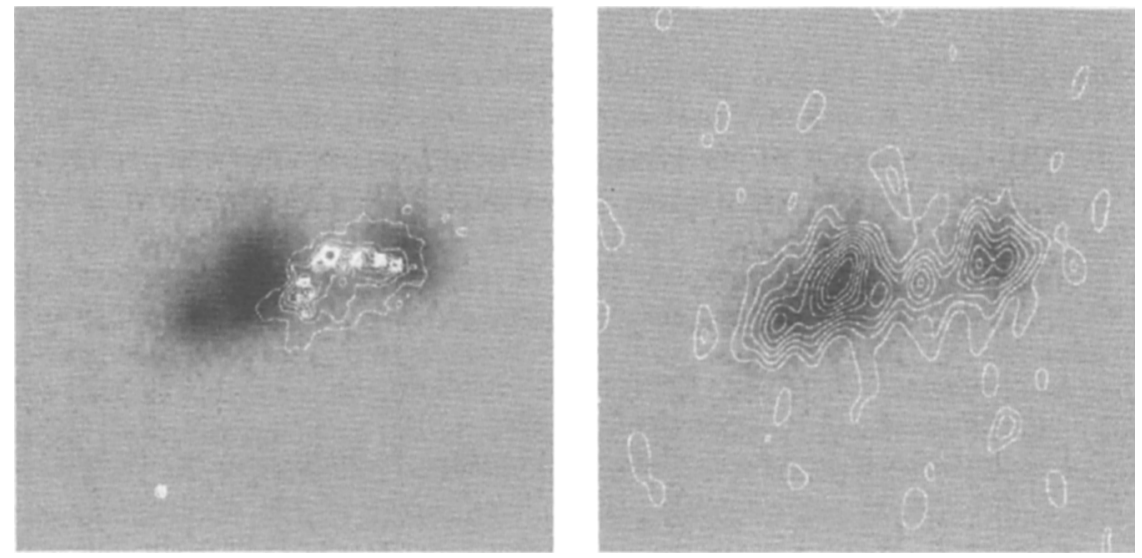

Figure 1. The center of He 2-10 as seen in the $N$-band (greyscale), the HST $V$-band (contours, left), and $2 \mathrm{~cm}$ radio (contours, right). The FoV is $9.4^{\prime \prime} \times 9.4^{\prime \prime}$, or $410 \mathrm{pc} \times 410 \mathrm{pc}$.

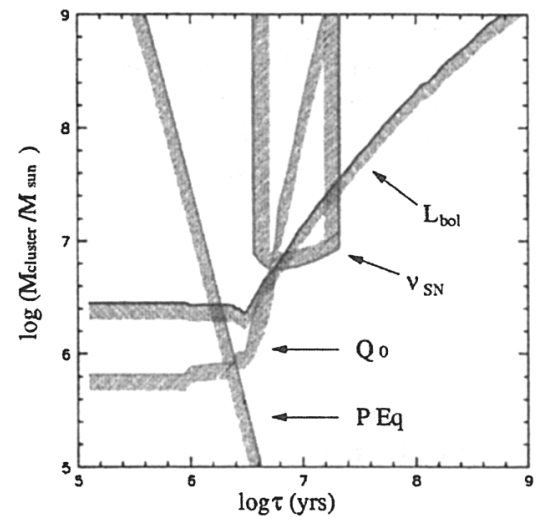

Figure 2. Constraints on $\tau$ and $M$ of the stellar cluster embedded in the brightest $N$-band knot detected in He 2-10. Regions of parameter space below the lines labeled $L_{\mathrm{bol}}, Q_{0}$, and $P_{E q}$ are excluded by the models, based on considerations of estimated $L_{\mathrm{bol}}$, $L_{\text {ion }}$, and the assumption of pressure equilibrium with the surrounding ISM. The region above the line labeled as $\nu_{\mathrm{SN}}$ is excluded based on the upper limit of non-thermal contributions to the flux from SNe. This cluster must have an age of $0.4<\tau<3.6 \mathrm{Myr}$ and a mass of $\mathrm{M}_{\odot}>2.5 \times 10^{6} \mathrm{M}_{\odot}$.

cantly larger than the average for normal galaxies, but comparable to those for ultraluminous IR galaxies. Large ratios for some starburst galaxies may therefore indicate, that a significant fraction of the far-IR flux may arise from UDH II regions. If most of the far-IR flux from $\mathrm{He} 2-10$ and other starbursts is produced by such heavily obscured regions, the well-known correlation between UV continuum slope and IR-to-UV flux ratio observed for starbursts (e.g., Meurer et al. 1995) cannot be due primarily to UV reprocessing by dust in a foreground screen, but must simply reflect a trend in the overal dust content in these galaxies. The dust that reddens the UV continuum must be largely decoupled from the dust that produces the far-IR flux in some starbursts.

\section{References}

Kobulnicky, H.A., Johnson, K.E. 1999, ApJ 527, 154

Meurer, G.R., Heckman, T.M., Leitherer, C., et al. 1995, AJ 110, 2665

Vacca, W.D., Johnson, K.E., Conti, P.S. 2002, AJ 123, 772 